\title{
Surfactant Mediated Heteroepitaxy versus Homoepitaxy: Kinetics for Group-IV Adatoms on As-Passivated $\mathrm{Si}(111)$ and $\mathrm{Ge}(111)$
}

\author{
K. Schroeder, A. Antons, R. Berger, and S. Blügel* \\ Institut für Festkörperforschung, Forschungszentrums Jülich, D-52425 Jülich, Germany
}

(Received 10 May 2001; published 4 January 2002)

\begin{abstract}
Using ab initio calculations we have determined the paths and activation energies for diffusion of group-IV atoms ( $\mathrm{Si}, \mathrm{Ge}$, and $\mathrm{Sn}$ ) on top of the As layer on As-passivated $\mathrm{Si}(111)$, and for exchange with an As atom. The kinetics of $\mathrm{Si}, \mathrm{Ge}$, and $\mathrm{Sn}$ adatoms is substantially different: $\mathrm{Si}$ adatoms are readily incorporated under the As layer. Ge adatoms diffuse far on top of the As layer and can reach existing steps. We show for the first time that the ratio between diffusion and exchange barriers depends strongly on the strain of the growing Ge film. Sn atoms remain on top of the As layer.
\end{abstract}

DOI: 10.1103/PhysRevLett.88.046101

PACS numbers: $68.35 . \mathrm{Fx}, 68.55 .-\mathrm{a}, 71.15 .-\mathrm{m}$

In recent years progress in information technology has been fueled by the advances in electronic materials. Typically, multicomponent films with increasing requirements on the control of growth on nanoscale dimensions are used. For many applications (transistors, lasers) the growth of atomically flat layered heterostructures is crucial. Because of lattice mismatch this is not easily realized. An important example is the $\mathrm{Ge} / \mathrm{Si}$ heterostructure whose lattice constants differ by $\approx 4 \%$. It was shown [1-5] that the introduction of surfactant layers modifies the growth mode of $\mathrm{Ge}$ on Si drastically. Whereas on the clean $\mathrm{Si}(111)$ surface Ge grows in the Stranski-Krastanov mode with large three-dimensional islands, with an overlayer of $\mathrm{As}, \mathrm{Sb}$, or $\mathrm{Bi}$ one finds layer-by-layer-growth on $\mathrm{Si}(111)$ and $\mathrm{Si}(001)$. A MOSFET structure with $p$-type $\mathrm{Ge}$ as the active layer has successfully been grown on a $\mathrm{Si}(111)$ substrate using an $\mathrm{Sb}$ surfactant layer [6]. Growth on the more common $\mathrm{Si}(001)$ substrates was not successful [7] because the appearance of "V-shaped defects" due to misfit dislocations destroys the Ge layers. A full microscopic explanation of the surfactant effect is still missing, but several aspects have been clarified: Because of the additional electron, surfactant layers of group-V elements on $\mathrm{Si}$ or Ge modify the reconstruction of the surface. For example, the As-covered $\mathrm{Si}(111)$ surface shows a $(1 \times 1)$ structure $[8]$ instead of the $(7 \times 7)$ reconstruction of pure $\mathrm{Si}(111)$. Group- $\mathrm{V}$ atoms have a reduced-surface free energy which makes them float on the surface without being incorporated into the growing crystal $[1,9]$. Thus, growth proceeds by incorporation of deposited $\mathrm{Si}$ or $\mathrm{Ge}$ atoms under the surfactant layer. In addition, the kinetics of deposited adatoms are changed by the presence of the surfactant layer. Kaxiras and Kandel [10-12] have argued in a series of papers that (i) due to the passivation of the surface the barrier for diffusion of adatoms should always be lower than for incorporation, (ii) surfactants not only passivate the flat surface but also the step edges, which reduces the incorporation probability of adatoms. Thus, in spite of a long diffusion length of adatoms homogeneous nucleation of islands on the terraces would be possible, as found experimentally. Experiments of Voigtländer et al. [13] on homoepitaxy on $\mathrm{Si}(111)$ show that the island density increases when surfactants (As or $\mathrm{Sb})$ are present. The authors interpreted this as a decrease of the effective diffusion constant due to surfactants. We have shown in a previous Letter [14] that for Si adatoms on $\mathrm{Si}(111)$ passivated by one monolayer (ML) As, Si(111):As, the exchange barrier for incorporation under the As layer is as low as the diffusion barrier, and $\mathrm{Si}$ atoms gain a substantial binding energy of $E_{B}=0.80 \mathrm{eV}$ when they are incorporated. Thus, the long range $\mathrm{Si}$ diffusion on $\mathrm{Si}(111)$ :As is reexchange dominated and governed by the reexchange barrier $E_{\text {reex }} \approx 1.1 \mathrm{eV}$. It is slowed down compared to the clean $\operatorname{Si}(111)(7 \times 7)$ surface, which is in full accord with the experiments.

The question is whether the Si results can be transferred to the case of Ge heteroepitaxy. Detailed experimental studies of Ge heteroepitaxy on $\mathrm{Si}(111): \mathrm{Sb}[15,16]$ reveal that growth proceeds in several steps. (i) A flat compressed Ge double layer (DL) is completed epitaxially underneath the surfactant layer; (ii) growth proceeds with a roughness of less than two layers until (iii) the Ge film releases strain and partially relaxes by the formation of misfit dislocations at the $\mathrm{Ge} / \mathrm{Si}$ interface after 10-12 layers of Ge are deposited; (iv) further Ge growth proceeds homoepitaxially on the relaxed $\mathrm{Ge}(111)$ :Sb film.

In the present Letter we investigate this scenario by reporting results on the kinetics of single Ge adatoms (i) on $\mathrm{Si}(111): \mathrm{As}$, (ii) on $\mathrm{Si}(111) / \mathrm{Ge}(\mathrm{DL}): \mathrm{As}$ at the $\mathrm{Si}$ lattice constant, and (iii) on $\mathrm{Ge}(111)$ :As with different lattice constants (ranging between the equilibrium lattice constants $a_{\mathrm{Si}}$ and $a_{\mathrm{Ge}}$ ) in order to simulate the role of strain of the growing and relaxing Ge film. We have chosen the As-covered systems since they show the simple $(1 \times 1)$ surface structure. This makes the calculations feasible using a Cray-T3E supercomputer. We have calculated the paths and energy barriers for group-IV atoms ( $\mathrm{Si}, \mathrm{Ge}$, and Sn) diffusion on top of the As layer and for the exchange of Ge with an As atom. The calculated energies are used to discuss the scenario for epitaxy. We find that the three considered adatoms behave quite differently. The relation 
between the diffusion and exchange barriers strongly depends on the type of adatom, on the substrate material, and on the surface strain.

All calculations have been carried out with a massively parallelized $a b$ initio molecular dynamics program (for details, see Ref. [17]), using norm-conserving pseudopotentials of the Kleinman-Bylander form [18] and the local density approximation [19]. Force calculations combined with molecular statics [20] are used to determine the minimum energy configurations and the reaction pathways. The systems are simulated by a repeated slab model consisting of six atomic $\mathrm{Si}(111)$ layers in an inversion symmetric arrangement, $1 \mathrm{ML}$ As on each Si surface, and one adatom on each As layer, separated by a vacuum equivalent to eight atomic Si layers. The same arrangement was chosen to simulate the Ge(111) surface, and the $\mathrm{Si} / \mathrm{Ge}(\mathrm{DL})$ system was modeled by a symmetric (111) oriented As: $\mathrm{Ge}(\mathrm{DL}) / \mathrm{Si}(\mathrm{DL}) / \mathrm{Ge}(\mathrm{DL})$ :As heterostructure. We find that a $(3 \times 3)$ periodic cell in the lateral directions, four $\mathbf{k}_{\|}$points in the surface Brillouin zone, and a cutoff energy of $13.69 \mathrm{Ry}$ in the plane wave basis set are necessary to obtain well converged results [21]. For all systems we applied the symmetry-unrestricted geometry optimization including all atoms except those of the two innermost atomic layers of the slab. To establish minimum energy configurations we converged the forces acting on the atoms to less than $0.1 \mathrm{mRy} /$ a.u.

The equilibrium adatom configurations are determined by minimizing the total energy of the supercell with respect to all atomic coordinates, starting from an arbitrary adatom position on top of the As layer. It turns out to be the H3 site (above the empty hexagon with three As neighbors in the top layer and three Si neighbors in the second layer; see Fig. 1a) for all group-IV adatoms. Saddle points are determined by restricted relaxation similar to the "hyperplane adaptive constraint" (HAC) method [22], i.e., by minimizing the energy subject to additional constraints. We find that the T4 site (above the position of

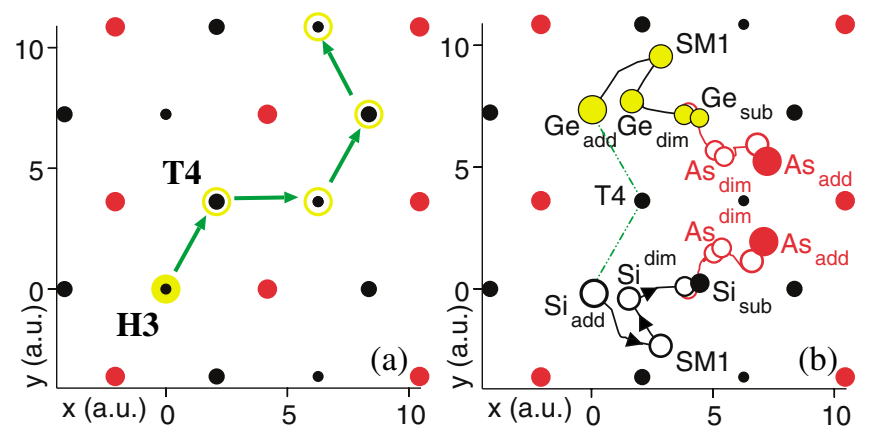

FIG. 1 (color). Top view of diffusion and exchange paths for group-IV atoms on $\mathrm{Si}(111)$ :As. (a) The equilibrium sites on top of the As layer are at $\mathrm{H} 3$ (small black dots), and the diffusion saddle points at T4 (medium black dots); As atoms are red dots. (b) The exchange paths for Si (lower half of figure) and Ge (yellow; upper half) adatoms are very similar. The exchanging pairs visit several local minima (open circles, whose size indicates the height above the surface). the second layer $\mathrm{Si}$ atoms) is the diffusion saddle for $\mathrm{Ge}$ and $\mathrm{Sn}$, as previously found for Si adatoms. The barriers for on-top diffusion, i.e., the energy differences between $\mathrm{H} 3$ and $\mathrm{T} 4, E_{D} \approx 0.25 \mathrm{eV}$ (see Table I), are very similar for all group-IV atoms, in spite of the different atom sizes and bond strengths. This verifies the effective passivation of the $\mathrm{Si}(111)$ surface by the As layer. A top view of the resulting diffusion path is shown in Fig. 1a.

The minimum energy configuration of incorporated group-IV atoms at the end of an exchange process consists of a substitutional group-IV atom replacing an As atom which becomes an adatom located at a bridge site binding to the substitutional $\mathrm{Si}(\mathrm{Ge}, \mathrm{Sn})$ atom and a neighboring As atom. As can be seen from Table I the energy gain drastically depends on the atom size. While the substitutional $\mathrm{Si}$ atom is bound with an additional energy of $E_{B}=-E_{\text {sub }}=0.80 \mathrm{eV}$, the $\mathrm{Ge}$ atom is bound only by about $0.20 \mathrm{eV}$, and the substitutional $\mathrm{Sn}$ atom has an even higher energy than the Sn adatom (negative binding energy) of $0.44 \mathrm{eV}$. Thus, $\mathrm{Sn}$ is not incorporated underneath the As layer, and is not further considered. This trend relates to the reduction of the covalent bond strength with increasing spatial extension of the $p$ states with an increasing number of nodes in relation to the size to the atom. Covalent $\mathrm{C}$ bonds in diamond are the strongest, and in Sn the weakest.

We find a low energy path for the exchange of a Ge-As pair which is very similar to the previously determined $\mathrm{Si}$ exchange path as can be seen in Fig. 1b. The exchange path is very complex and consists of several sections between local minima: First, the exchanging Ge adatom moves partially towards the diffusion saddle point. Then, it dips down towards the Si layer, pushing the As atom out of its substitutional position. From this first local minimum position "SM1" the Ge-As pair proceeds to the "dimer" position where the two atoms form a molecule lying parallel to the surface. On the final section, the Ge atom moves to the substitutional position while the As atom moves upward to its equilibrium adatom bridge site. We have determined the saddle points between all local minima. Even though the paths are similar for $\mathrm{Si}$ and $\mathrm{Ge}$, the energies on the paths differ substantially, as can be seen in Fig. 2 . For Si the highest energy is found on the first section, and $E_{\text {ex }}(\mathrm{Si})=E_{\mathrm{ex}}^{(1)}=0.27 \mathrm{eV}$ is comparable to $E_{D}(\mathrm{Si})$. When the Ge atom approaches the Si layer the energy rises above that of a Si adatom. On this last section of the path the energy for Ge-As exchange is shifted upward almost rigidly by $\sim 0.5 \mathrm{eV}$ compared to the $\mathrm{Si}-\mathrm{As}$ exchange, nearly the

TABLE I. Energies (in eV; relative to the $\mathrm{H} 3$ adatom position) for group-IV adatoms on $\mathrm{Si}(111)$ :As in different configurations.

\begin{tabular}{crrr}
\hline \hline Conf. & Si & Ge & Sn \\
\hline$E_{D}$ & 0.25 & 0.25 & 0.23 \\
$E_{\text {ex }}$ & 0.27 & 0.71 & \\
$E_{\text {sub }}=-E_{B}$ & -0.80 & -0.20 & 0.44 \\
$E_{\text {reex }}$ & 1.07 & 0.91 & \\
\hline \hline
\end{tabular}




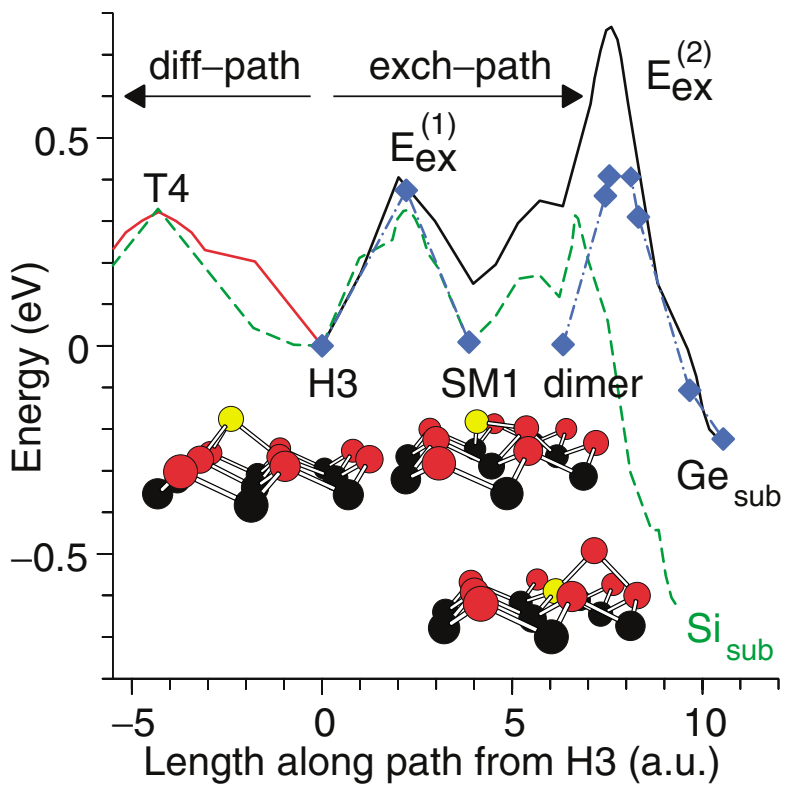

FIG. 2 (color). Energy (relative to the H3 position of the adatom; for $E_{\text {cut }}=9 \mathrm{Ry}$ ) along the diffusion path (to the left of $\mathrm{H} 3$ ) and along the exchange path to the substitutional configuration (to the right). Compared are the energies for $\mathrm{Si}$ (dashed line) and $\mathrm{Ge}$ (full line) on $\mathrm{Si}(111)$ at $a_{\mathrm{Si}}$, and for $\mathrm{Ge}$ on $\mathrm{Ge}(111)$ at $a_{\mathrm{Ge}}$ (diamonds). The atomic configurations for important minima are shown as insets: H3: adatom equilibrium; Ge-As dimer; $\mathrm{Si}(\mathrm{Ge})_{\text {sub }}$ : substitutional $\mathrm{Si}(\mathrm{Ge})$ and As adatom [yellow: $\mathrm{Si}(\mathrm{Ge})$ adatom; red: As; black: Si bulk].

same amount by which the binding energies of the incorporated atoms differ. Apparently, the bond strength argument is already important when the Ge atom approaches the Si layer. For Ge the highest saddle point is found on the last section, and $E_{\mathrm{ex}}(\mathrm{Ge})=E_{\mathrm{ex}}^{(2)}=0.70 \mathrm{eV}$ is much larger than $E_{D}(\mathrm{Ge})$ [23]. This value for the exchange energy is somewhat less than the estimate of $0.8 \mathrm{eV}$ by Kandel and Kaxiras [11] for the exchange of a full Ge layer on $\mathrm{Si}(111) / \mathrm{Ge}(\mathrm{DL}): \mathrm{Sb}$. We suggest that the subtle balance between the bond energies of $\mathrm{Si}-\mathrm{Ge}$ and $\mathrm{Si}-\mathrm{As}$ pairs aided by some extra strain energy when a bigger Ge atom is squeezed into the As layer at the lattice constant of $\mathrm{Si}$ is responsible for the high $\mathrm{Ge}$ exchange energy barrier. To substantiate this point we have calculated the Ge exchange path for As-covered $\mathrm{Ge}(111)$ (at the Ge equilibrium lattice constant) and indeed find the low value $E_{\mathrm{ex}}(\mathrm{Ge})=0.40 \mathrm{eV}$ (see the diamonds in Fig. 2).

The relation between the energy barriers has consequences for the diffusion behavior and growth mode, and we suggest the following scenario: (i) As discussed above, the long range diffusion of Si adatoms on $\mathrm{Si}(111)$ :As is dominated by the reexchange process, and the effective diffusion barrier is the reexchange energy: $E_{D \text {,eff }}=E_{\text {reex }}=$ $1.1 \mathrm{eV}$. (ii) On substrates with the Si lattice constant $\mathrm{Ge}$ adatoms behave very differently from Si. Since the exchange energy $E_{\mathrm{ex}}(\mathrm{Ge})=0.7 \mathrm{eV}$ is much higher than the diffusion barrier $E_{D}(\mathrm{Ge})=0.25 \mathrm{eV}, \mathrm{Ge}$ adatoms perform a large number of diffusion jumps, $n_{D}$, before they are incorporated under the As layer on $\mathrm{Si}(111)$. One can estimate that at a typical growth temperature of $600{ }^{\circ} \mathrm{C}, n_{D} \approx 500$ [with the higher $E_{\mathrm{ex}}(\mathrm{Ge})$ on strained Ge films (see below) $n_{D}$ is even 5000], and thus Ge adatoms have a good chance to visit terrace steps. This would suggest that Ge heteroepitaxy on $\mathrm{Si}(111)$ :As proceeds by step flow. But, following Kandel and Kaxiras [11], we may speculate that all dangling bonds of $\mathrm{Si}$ at the step edge are saturated by attached As atoms. One then may expect a similar attachment process at the step edge as for exchange on the flat terrace, and a competition of island nucleation and step flow results.

To get insight into the adatom kinetics on the growing and strain relaxing Ge film we have calculated the relevant equilibrium positions and energy barriers for a Ge adatom on epitaxial $\mathrm{Si}(111) / \mathrm{Ge}(\mathrm{DL})$ :As (at the Si lattice constant) and on Ge(111):As for different lateral lattice constants. The results are summarized in Table II and Fig. 3. For all systems at the lattice constant of $\mathrm{Si}$ we find the diffusion barrier of $E_{D}(\mathrm{Ge}) \approx 0.25 \mathrm{eV}$ and a large exchange barrier irrespective of the substrate $[\mathrm{Si}, \mathrm{Si} / \mathrm{Ge}(\mathrm{DL})$, or strained $\mathrm{Ge}$. With decreasing strain, i.e., increasing lateral lattice constant, we find a very pronounced decrease of the exchange barriers, whereas the diffusion barrier stays almost constant. This is in contrast to the behavior on metals, where due to the increasing electron-density corrugation an increase of the diffusion barrier is expected with expanding lattice constant [24]. We find that the ratio $E_{\text {ex }}(\mathrm{Ge}) / E_{D}$ and the reexchange energy $E_{\text {reex }}$ decrease when the Ge film relaxes. The situation at the equilibrium lattice constant of $\mathrm{Ge}$ is similar to homoepitaxy of $\mathrm{Si}$ on $\mathrm{Si}(111)$ :As; diffusion and exchange barriers are comparable. We have also considered a $2 \%$ dilated $\mathrm{Ge}(111)$ :As film which can be realized on substrates with a larger lattice constant than Ge. As seen in Fig. 3, the exchange barriers decrease further. In addition, the most stable position of Ge changes from the substitutional site to the dimer configuration.

In summary, our calculations suggest that no conclusions on heteroepitaxy can be drawn from the results for homoepitaxy. For $\mathrm{Si}, \mathrm{Ge}$, and $\mathrm{Sn}$ adatoms on As-covered $\mathrm{Si}(111)$ we have calculated the equilibrium configurations, the barriers for diffusion and for exchange with an As atom, and the binding energies of the incorporated atoms. The results can be understood on the basis of the different bond strengths due to the different sizes of the atoms.

TABLE II. Energies (in $\mathrm{eV}$; relative to the $\mathrm{H} 3$ adatom position for the respective system) of $\mathrm{Ge}$ adatoms on different films: $\mathrm{Si}(111)$ :As, $\mathrm{Si}(111) / \mathrm{Ge}(\mathrm{DL}): \mathrm{As}$, and $\mathrm{Ge}(111)$ :As at different lattice constants.

\begin{tabular}{crrrrrr}
\hline \hline $\begin{array}{c}\text { substr.: } \\
\text { strain: }\end{array}$ & \multicolumn{2}{c}{$\mathrm{Si} \mathrm{Si} / \mathrm{Ge}(\mathrm{DL})$} & \multicolumn{4}{c}{ Ge film } \\
\hline$E_{D}$ & 0.25 & 0.24 & 0.28 & 0.31 & 0.26 & 0.15 \\
$E_{\mathrm{ex}}^{(1)}$ & 0.41 & & 0.48 & & 0.37 & 0.18 \\
$E_{\mathrm{ex}}^{(2)}$ & 0.71 & 0.92 & 0.90 & 0.68 & 0.40 & 0.12 \\
$E_{\mathrm{dim}}$ & 0.23 & 0.37 & 0.34 & 0.09 & -0.12 & -0.29 \\
$E_{\text {sub }}$ & -0.20 & -0.18 & -0.21 & -0.19 & -0.12 & -0.06 \\
$E_{\text {reex }}$ & 0.91 & 1.09 & 1.11 & 0.87 & 0.53 & 0.46 \\
\hline \hline
\end{tabular}




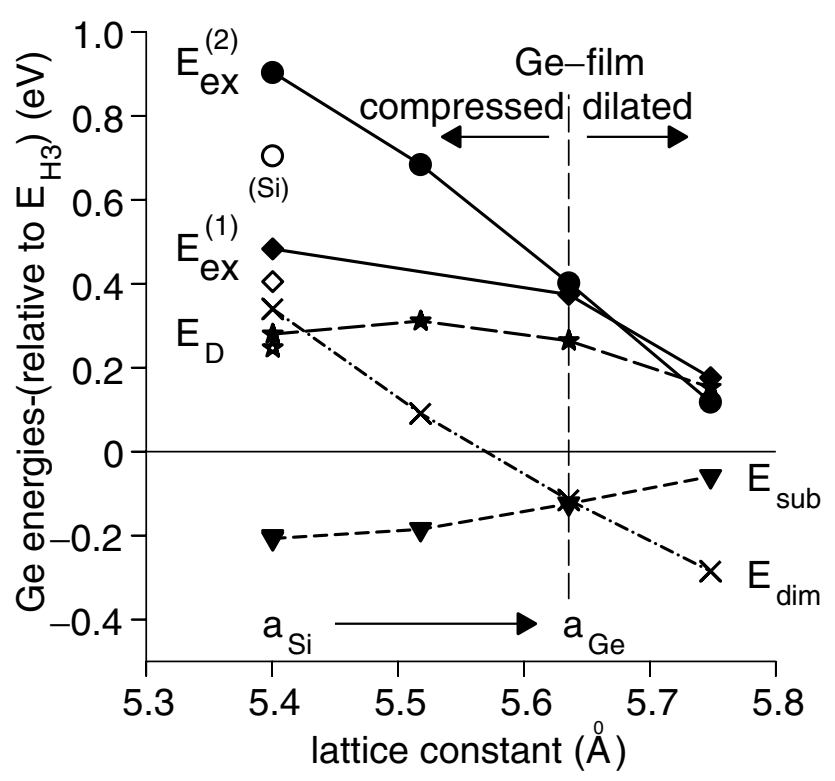

FIG. 3. Energies of minima and saddle points (relative to the $\mathrm{H} 3$ position) for Ge adatoms on $\mathrm{Ge}(111)$ :As versus the lattice constant [full symbols; open symbols: $\mathrm{Si}(111)$ :As]. Plotted are the exchange barriers, $E_{\mathrm{ex}}^{(1)}$ (dots connected by full line) and $E_{\mathrm{ex}}^{(2)}$ (diamonds and dash-3-dotted line), the diffusion barrier $E_{D}$ (stars and long-dashed line), the substitutional energy $E_{\text {sub }}$ (triangles and dashed line), and the energy of the intermediary dimer position $E_{\mathrm{dim}}$ (crosses and dash-dotted line).

Homoepitaxy and heteroepitaxy on $\mathrm{Si}(111)$ :As proceed by very different mechanisms: (i) $\mathrm{Si}$ atoms are readily incorporated and homoepitaxy proceeds exclusively via nucleation and growth of two-dimensional $\mathrm{Si}$ islands on the terraces. (ii) $\mathrm{Ge}$ atoms on $\mathrm{Si}$ :As or Ge:As films with the Si lattice constant have to overcome a large exchange barrier and show a moderate binding to the substitutional site. Growth can proceed by a mixture of step flow and island nucleation on the terrace, depending on the unknown efficiency of As passivation of steps. The exchange probability of a Ge adatom increases when the Ge film grows and relaxes; for a fully relaxed Ge film at the equilibrium lattice constant of Ge we find a similar situation as for homoepitaxy of Si. (iii) $\mathrm{Sn}$ atoms are not incorporated but grow on top of As.

This work was supported by the BMBF Verbundprojekt, Project No. 22.01D.

*Permanent address: Fachbereich Physik, Universität Osnabrück, D-49069 Osnabrück, Germany.

[1] M. Copel, M. C. Reuter, E. Kaxiras, and R. M. Tromp, Phys. Rev. Lett. 63, 632 (1989).
[2] M. Horn-vonHoegen et al., Phys. Rev. Lett. 67, 1130 (1991).

[3] G. Meyer, B. Voigtländer, and N. M. Amer, Surf. Sci. 274, L541 (1992).

[4] B. Voigtländer and A. Zinner, J. Vac. Sci. Technol. A 12, 1932 (1994).

[5] T. Schmidt et al., Appl. Phys. Lett. 74, 1391 (1999).

[6] D. Reinking et al., Electron. Lett. 35, 503 (1999).

[7] F. K. LeGoues, M. Copel, and R. M. Tromp, Phys. Rev. B 42, 11690 (1990).

[8] M. A. Olmstead, R. D. Bringans, R. I. G. Uhrberg, and R. Z. Bachrach, Phys. Rev. B 34, 6041 (1986).

[9] S. Oppo, V. Fiorentini, and M. Scheffler, Phys. Rev. Lett. 71, 2437 (1993).

[10] E. Kaxiras, Mater. Sci. Eng. B 30, 175 (1995).

[11] D. Kandel and E. Kaxiras, Phys. Rev. Lett. 75, 2742 (1995).

[12] D. Kandel, Phys. Rev. Lett. 78, 499 (1997).

[13] B. Voigtländer, A. Zinner, Th. Weber, and H. P. Bonzel, Phys. Rev. B 51, 7583 (1995).

[14] K. Schroeder, B. Engels, P. Richard, and S. Blügel, Phys. Rev. Lett. 80, 2873 (1998).

[15] M. Horn-von Hoegen, Z. Kristallogr. 214, 591 (1999); 214, 684 (1999).

[16] N. Theuerkauf, Ph.D. thesis, RWTH Aachen, 1999.

[17] R. Berger et al., in Proceedings of the Workshop on Molecular Dynamics on Parallel Computers, Jülich, Germany, 1999 (World Scientific, Singapore, River Edge, NJ, 2000), p. 185.

[18] L. Kleinman and D. M. Bylander, Phys. Rev. Lett. 48, 1425 (1982).

[19] S. H. Vosko, L. Wilk, and M. Nusair, Can. J. Phys. 58, 1200 (1980).

[20] R. Fletcher, Practical Methods of Optimization (John Wiley \& Sons, New York, 1987).

[21] The total energies for minima and saddle points were calculated with cutoff energies of 13.69 and 9 Ry, using four and nine $\mathbf{k}_{\|}$points, as well as for thicker slabs of 12 layers. The atomic configurations do not change much with the size of the basis set $(\Delta d<0.05 \AA)$. The estimated error of energy differences is approximately $0.05 \mathrm{eV}$. The values quoted in the tables are for 13.69 Ry cutoff and four $\mathbf{k}_{\|}$points; for the determination of the entire paths shown in Figs. $1 \mathrm{~b}$ and 2 we used 9 Ry.

[22] J. J. Mortensen, Y. Morikawa, B. Hammer, and J. K. Norskov, J. Catal. 169, 85 (1997).

[23] Our results are in striking discrepancy to the findings of Ko [Y.-J. Ko, K. J. Chang, and J.-Y. Yi, Phys. Rev. B 60, 1777 (1999)], who report a low energy barrier of $0.2 \mathrm{eV}$ for the Ge-As-exchange. We essentially rediscovered our exchange path described above, when we tried to recalculate their transition path from the Ge adatom position at a bridge site to the substitutional position of the incorporated $\mathrm{Ge}$ atom.

[24] B. D. Yu and M. Scheffler, Phys. Rev. B 56, R15569 (1997). 